\title{
SOME DISTORTION THEOREMS FOR MULTIVALENT MAPPINGS
}

\author{
H. J. LANDAU AND R. OSSERMAN ${ }^{1}$
}

The following lemma was first considered by Ahlfors [1] and later by Polya [3], both of whom gave simple proofs and quite different applications. Later Jenkins [2] gave a considerably more involved proof whose merit was its generalizability to other problems. In this note we introduce two methods which yield simple proofs of all of the results in [2] and lead to still further interesting generalizations.

Lemma. Let $R$ be the rectangle $|x|<a,|y|<b$ of the z-plane, $z$ $=x+i y$. Let $w=f(z)$ be analytic in $R$ and continuous in the closure of $R$. With $w=u+i v$, assume

$$
\begin{array}{rlrl}
|v| & \leqq b^{\prime} \quad \text { for }|y| & =b, \\
u & \geqq a^{\prime}>0 \text { for } & x & =a, \\
u & \leqq-a^{\prime} \quad \text { for } & x & =-a .
\end{array}
$$

Then $b / a \leqq b^{\prime} / a^{\prime}$. Equality can occur only if $f(z)=\left(a^{\prime} / a\right) z$.

Proof. Over each horizontal segment of $R$ we have

$$
\int \frac{\partial u}{\partial x} d x \geqq 2 a^{\prime}
$$

Hence

$$
\iint_{R} \frac{\partial u}{\partial x} d x d y \geqq 2 a^{\prime} b .
$$

On each vertical segment

$$
\int \frac{\partial v}{\partial y} d y \leqq 2 b^{\prime}
$$

so that

$$
\iint_{R} \frac{\partial v}{\partial y} d x d y \leqq 2 a b^{\prime} .
$$

Since $\partial u / \partial x=\partial v / \partial y$ throughout $R$, the result follows.

Received by the editors March 28, 1958 and, in revised form, April 28, 1958 and June 6, 1958.

1 This paper was written while the second author was a Temporary Member of the Institute of Mathematical Sciences, New York University. 
For equality to hold it must hold at each step which would imply that the sides of $R$ must map into the corresponding sides of the rectangle $R^{\prime}:|u|<a^{\prime},|v|<b^{\prime}$. Then by the argument principle the map is one-to-one, and since the vertices correspond, the map is uniquely determined, hence of the form indicated.

A curious feature of the above proof is the very weak way in which analyticity is used. In fact the proof can be applied without change to show the following:

TheOREM 1. If instead of analyticity of $f(z)$ in the above lemma we merely assume that $\partial u / \partial x$ and $\partial v / \partial y$ are continuous and equal, ${ }^{2}$ then the inequality still holds.

Of course in this case we cannot assert uniqueness.

The application made by Polya to a generalization of a wellknown theorem of Lindelöf also carries over. We reproduce the proof for this case.

THEOREm 2. Let $S$ be the semi-infinite strip $a<x<b, y>0$. Suppose $u$ and $v$ are continuously differentiable in $S$, continuous in the closure, and satisfy $\partial u / \partial x=\partial v / \partial y$. If there exist real numbers $A, B$ with $A<B$ and $y_{0}>0$ such that $u \leqq A$ for $x=a, y \geqq y_{0}$, and $u \geqq B$ for $x=b, y \geqq y_{0}$, then $v$ cannot remain bounded in $S$.

In particular, if $f(z)$ is analytic in $S$ and approaches distinct limits on the vertical sides as $y$ tends to infinity, then for some real $\alpha$, the functions $u=\operatorname{Re}\left\{e^{i \alpha} f(z)\right\}$ and $v=\operatorname{Im}\left\{e^{i \alpha} f(z)\right\}$ satisfy the hypotheses, and hence $|f(z)|$ cannot be bounded, which is Lindelöf's theorem.

Proof. Let $m$ be the minimum of $v$ for $y=y_{0}$, and $M$ the maximum of $v$ for $y=y_{1}$ for any $y_{1}>y_{0}$. Then by a translation in both planes we can apply Theorem 1 to the rectangle bounded by $x=a, x=b$ and $y=y_{0}, y=y_{1}$, giving

$$
\frac{y_{1}-y_{0}}{b-a} \leqq \frac{M-m}{B-A} .
$$

Since $y_{1}$ may be chosen arbitrarily large, the result is proved.

The method used to prove the lemma can, like that of Jenkins, be applied to a theorem which in the plane case is due to Schiffer. ${ }^{3}$

TheOREM 3. Let $W_{i}, i=1,2$, be arbitrary Riemann surfaces. Let $R_{i}$ be a relatively compact region on $W_{i}$ bounded by a finite number of Jor-

2 The referee has kindly pointed out that in the proofs of Theorems 1 and 2 all that is used is the inequality $\partial u / \partial x \leqq \partial v / \partial y$.

${ }^{3}$ Oral communication. 
dan curves and let $C_{i}$ be a distinguished boundary curve of $R_{i}$. Let $u_{i}$ be the harmonic measure of $C_{i}$ with respect to $R_{i}$, and let $p_{i}$ be the period around $C_{i}$ of the harmonic conjugate to $u_{i}$. If $f(p)$ is an analytic map of $R_{1}$ into $R_{2}$ and if for some (and hence every) curve $C_{1}^{*}$ homologous to $C_{1}$, the image curve $f\left(C_{1}^{*}\right)$ is homologous to $n C_{2}$ for some integer $n$, then $p_{1} \geqq|n| p_{2}$.

Rather than give a detailed proof by the above method, we merely note that by completing $u_{i}$ to an analytic function we obtain a map of $R_{i}$ with certain cuts onto a rectangle of width 1 and height $p_{i}$, cut along horizontal lines, and applying a slight modification of the procedure used in the lemma to these rectangles gives the result. We give instead another proof from which one can derive considerably more information.

Proof. We first carry out the proof for the case where the boundary curves of $R_{1}$ are analytic, $f$ is analytic in the closure $\bar{R}_{1}$ of $R_{1}$ and maps $\bar{R}_{1}$ into $R_{2}$. By means of the map $f(p)$ the function $u_{2}$ can then be carried back and considered as a harmonic function in $\bar{R}_{1}$. By the reflection principle $u_{1}$ is harmonic in $\bar{R}_{1}$, and hence also the function $v=u_{1}-u_{2}$. Denoting by $C$ the union of the boundary curves of $R_{1}$ other than $C_{1}$, we may define a function $w$ to be harmonic in $R_{1}$ and to satisfy $w=v$ on $C_{1}, w=0$ on $C$. Again by reflection $w$ is harmonic on $C$ and $w-v$ on $C_{1}$. Since $v$ also is harmonic on $C_{1}$, so is $w$. Thus all the functions $u_{1}, u_{2}, v, w$ are harmonic in $\bar{R}_{1}$ and have normal derivatives on the boundary. We have further on $C_{1}: u_{1}=1, u_{2} \leqq 1, v \geqq 0$, $w \geqq 0$, while on $C: u_{1}=0, u_{2} \geqq 0, v \leqq 0, w=0$. By the maximum principle $w \geqq 0$ in $R_{1}$. Using $\partial / \partial n$ to denote the derivative with respect to the exterior normal, we have therefore $\partial w / \partial n \leqq 0$ on $C$. Similarly $\partial(v-w) / \partial n \geqq 0$ on $C_{1}$. Hence

$$
\int_{C_{1}} \frac{\partial v}{\partial n} d s \geqq \int_{C_{1}} \frac{\partial w}{\partial n} d s=-\int_{C} \frac{\partial w}{\partial n} d s \geqq 0,
$$

and

$$
p_{1}=\int_{C_{1}} \frac{\partial u_{1}}{\partial n} d s \geqq \int_{C_{1}} \frac{\partial u_{2}}{\partial n} d s=\int_{f\left(C_{1}\right)} \frac{\partial u_{2}}{\partial n} d s=n p_{2} .
$$

In the above reasoning the only property of $u_{2}$ which we used was $0 \leqq u_{2} \leqq 1$. Therefore if we replace $u_{2}$ by $u_{2}^{\prime}=1-u_{2}$, we obtain

$$
p_{1} \geqq \int_{f\left(C_{1}\right)} \frac{\partial u_{2}^{\prime}}{\partial n} d s=-\int_{f\left(C_{1}\right)} \frac{\partial u_{2}}{\partial n} d s=-n p_{2} .
$$

Combining these two inequalities gives $p_{1} \geqq|n| p_{2}$. 
To obtain the result for the general case, we note that, for any $\delta>0$, the set $\delta \leqq u_{1} \leqq 1-\delta$ is a closed subset of the compact domain $\bar{R}_{1}$, and hence is compact. Thus it can contain only a finite number of critical points of $u_{1}$, from which it follows that there exist level curves $C^{\prime}: u_{1}=\epsilon$ and $C_{1}^{\prime}: u_{1}=1-\epsilon$, with $\epsilon$ arbitrarily small, on which the gradient of $u_{1}$ is never zero and which are therefore analytic. Let $R_{1}^{\prime}$ be the set of points in $R_{1}$ where $\epsilon<u_{1}<1-\epsilon$. The function $u_{1}^{\prime}$ $=\left(u_{1}-\epsilon\right) /(1-2 \epsilon)$ is equal to 1 on $C_{1}^{\prime}$, equal to 0 on $C^{\prime}$ and is harmonic in $\bar{R}_{1}^{\prime}$. The locus $C_{1}^{\prime}$ is homologous to $C_{1}$ since together they bound the region $1-\epsilon<u_{1}<1$, and hence $f$ maps $\bar{R}_{1}^{\prime}$ into $R_{2}$ with $f\left(C_{1}^{\prime}\right)$ homologous to $n C_{2}$. In this case neither $R_{1}^{\prime}$ nor $C_{1}^{\prime}$ need be connected, but $R_{1}^{\prime}$ is the union of a finite number of regions whose total boundary is $C_{1}^{\prime} \cup C^{\prime}$ and the reasoning used above may be applied precisely as before to yield

$$
\int_{c_{1}^{\prime}} \frac{\partial u_{1}^{\prime}}{\partial n} d s \geqq|n| p_{2} .
$$

But

$$
\frac{\partial u_{1}}{\partial n}=(1-2 \epsilon) \frac{\partial u_{1}^{\prime}}{\partial n}
$$

on $C_{1}^{\prime}$, and hence

$$
p_{1}=\int_{C_{1}^{\prime}} \frac{\partial u_{1}}{\partial n} d s \geqq(1-2 \epsilon)|n| p_{2} .
$$

Since this holds for arbitrarily small $\epsilon>0$, the result follows.

There are several generalizations of Theorem 3 which require no change in the proof, but only an elaboration of the statement. For example if we assume merely that each boundary component of $R_{i}$ is an arbitrary continuum containing at least two points, then the harmonic measures still exist, their level curves are analytic curves up to isolated singularities, and the above argument holds without change. We can no longer speak of $C_{1}^{*}$ and $f\left(C_{1}^{*}\right)$ as homologous to certain boundary curves, but we may, for example, require them to be homologous to some level curves of a harmonic measure and consider the periods around these level curves. This in fact also allows us to extend the result to the case that $R_{i}$ has an infinite number of boundary components. A detailed treatment of these and related questions will be given in a longer paper now under preparation.

Other results may be obtained by choosing curves homologous to a combination of boundary curves. Changing notation, let the two 
regions be $R$ and $R^{\prime}$ with boundary curves $C_{i}, i=1, \cdots, n$, and $C_{i}^{\prime}, i=1, \cdots, m$. Let $u_{i}$ be the harmonic measure of $C_{i}$ with respect to $R$, and let $p_{i j}$ be the period around $C_{i}$ of the conjugate to $u_{j}$. Define $u_{i}^{\prime}$ and $p_{i j}^{\prime}$ similarly with respect to $R^{\prime}$. We then have the following result:

If a curve $C$ in $R$ is homologous to $\sum_{i=1}^{r} C_{i}, r<n$, and if the image curve $f(C)$ in $R^{\prime}$ is homologous to $\sum_{i=1}^{s} n_{i} C_{i}^{\prime}, s<m$, for some integers $n_{i}$, then

$$
\sum_{i, j=1}^{r} p_{i j} \geqq\left|\sum_{i, j} n_{i} p_{i j}^{\prime}\right|,
$$

where, in the summation on the right-hand side, $i$ runs from 1 to $s$, and $j$ runs through an arbitrary subset $J$ of the integers from 1 to $\mathrm{m}$.

To see this we need only observe that the harmonic measure of the curves $\bigcup_{i=1}^{r} C_{i}$ with respect to $R$ is given by $u=\sum_{i=1}^{r} u_{i}$, whereas the function $u^{\prime}=\sum_{j \in J} u_{j}^{\prime}$ will be the harmonic measure of some set of boundary curves in $R^{\prime}$. Hence $0 \leqq u^{\prime} \leqq 1$ and if we set $v=u-u^{\prime}$ the proof follows exactly as in Theorem 3 .

A special feature of all the above distortion theorems, in contrast to most of those in conformal mapping, is that we never assume the functions to be one-to-one. In particular, Theorem 3 reduces to a particularly useful one in the doubly-connected case. There we may introduce as a conformal invariant the module $\mu=r_{2} / r_{1}$ where the domain is conformally equivalent to the annulus $r_{1}<|z|<r_{2}$. We then have $p_{i}=2 \pi / \log \mu_{i}$ and hence:

Corollary. If the doubly-connected domain $R_{i}$ has module $\mu_{i}$ and $R_{1}$ is mapped analytically into $R_{2}$ in such a way that the image of $C_{1}$ is homologous to $n C_{2}$, then $\mu_{2} \geqq \mu_{1}^{n}$.

This result was first proved and applied by Schiffer [4]. For references to other applications see the paper of Jenkins [2].

\section{REFERENCES}

1. L. Ahlfors, Sur une généralisation du théorème de Picard, C. R. Acad. Sci. Paris vol. 194 (1932) pp. 245-247.

2. J. Jenkins, Some results related to extremal length, Annals of Mathematics Studies, no. 30, (1953), pp. 87-94.

3. G. Polya, Über analytische Deformationen eines Rechtecks, Ann. of Math. vol. 34 (1933) pp. 617-620.

4. M. Schiffer, On the modulus of doubly-connected domains, Quart. J. Math. vol. 17 (1946) pp. 197-213.

The Bell Telephone laboratories, Murray Hill, and

STANFORD UNIVERSITY 